\title{
Non-Representability of Cohomology Classes by Bi-Invariant Forms (Gauge and Kac-Moody Groups)
}

\author{
Shrawan Kumar \\ Massachusetts Institute of Technology, Cambridge, MA 02139, USA and \\ Tata Institute of Fundamental Research, Colaba, Bombay 400005 , India
}

\begin{abstract}
We give a necessary topological condition on a cohomology class of any Lie group $\mathscr{G}$, modelled on a Fréchet space, to be representable by a biinvariant form on $\mathscr{G}$. As a corollary, we show that if $\Pi_{2 d}(\mathscr{G}) \underset{\mathbb{Z}}{\bigotimes} \mathbb{R} \neq 0$ for some $d>0$, then there exists a cohomology class in $H^{2 d}(\mathscr{G}, \mathbb{R})$ which cannot be represented by any bi-invariant form. In particular, we conclude that there are 'many' cohomology generators, in general, in the case of gauge groups and also Kac-Moody groups which cannot be represented by bi-invariant forms, although, very often, they are representable by left invariant forms.
\end{abstract}

\section{Introduction}

Using a mixture of (very simple) topological and geometrical arguments, we show that certain cohomology classes of infinite-dimensional Lie groups (modelled on Fréchet spaces) cannot be represented by bi-invariant forms.

Our main (and the only) theorem gives a necessary topological condition on a cohomology class, of a fairly arbitrary infinite dimensional group $\mathscr{G}$, to be representable by bi-invariant forms. An interesting corollary of the theorem is that if $x \in H^{2 d}(\mathscr{G}, \mathbb{R})$, with $d>0$ and $x$ is not decomposable (i.e. $x \notin H^{+}(\mathscr{G}, \mathbb{R}) \cdot H^{+}(\mathscr{G}, \mathbb{R})$ ) (such a $x$ always exists if $\Pi_{2 d}(\mathscr{G}) \bigotimes \mathbb{R} \neq 0$ ) then $x$ cannot be represented by biinvariant forms.

We apply this corollary to the particular (and important) examples of based loop groups, gauge groups and Kac-Moody groups to conclude that these groups, often, have many cohomology generators which cannot be represented by bi-invariant forms, although, in many cases, they can be represented by left invariant forms.

1. Definition. Let $M$ be a smooth $\left(=C^{\infty}\right)$ Fréchet manifold $[\mathrm{M}]$. By $\Delta_{\infty}(M)$, we mean

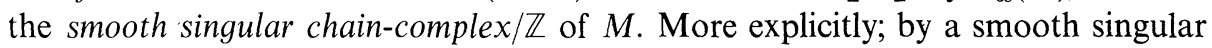
$n$-simplex in $M$, we mean a continuous map $s: \Delta^{n}=\left\{\left(t_{1}, \ldots, t_{n}\right) \in \mathbb{R}^{n}: t_{i} \geqq 0\right.$ and 
$\left.\sum_{i=1}^{n} t_{i} \leqq 1\right\} \rightarrow M$, such that $s$ extends to a smooth map on an open neighborhood of $\Delta^{n}\left(\right.$ in $\left.\mathbb{R}^{n}\right)$. We put $\Delta_{\infty}(M)=\sum_{n \geqq 0} \Delta_{\infty}^{n}(M)$, where $\Delta_{\infty}^{n}(M)$ is free abelian group on the set of all the smooth singular $n$-simplexes in $M$. We further define the smooth singular co-chain complex $\left(\right.$ of $M$ ) by $C_{\infty}(M, \mathbb{R})=\sum_{n \geqq 0} \operatorname{Hom}_{\mathbb{Z}}\left(\Delta_{\infty}^{n}(M), \mathbb{R}\right)$.

2. Lemma. Let $\mathscr{G}$ be a connected Lie group, modelled on a Fréchet space, (i.e., $\mathscr{G}$ is a paracompact, Hausdorff topological group with a $C^{\infty}$-Fréchet manifold structure on it such that the map $\mathscr{G} \times \mathscr{G} \rightarrow \mathscr{G}$, defined by $\left(g_{1}, g_{2}\right) \rightarrow g_{1} g_{2}^{-1}$, is smooth) with Lie algebra $\mathscr{L}(\mathscr{G})=\mathscr{L}$, and let $t: \mathscr{G} \rightarrow \mathscr{G}$ be the inversion map, defined by $t(g)=g^{-1}$. Then, we have the following commutative diagram:

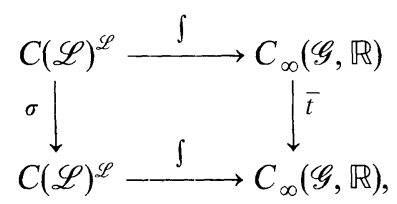

where $C(\mathscr{L})$ denotes the continuous co-chain complex of the Lie algebra $\mathscr{L}, C(\mathscr{L})^{\mathscr{L}}$ denotes the $\mathscr{L}$ invariants in $C(\mathscr{L})$ under the adjoint representation, $\sigma$ is the map which multiplies a homogeneous form of degree $n$ by $(-1)^{n}, \bar{t}$ is the map induced by $t$ and $\int$ is the integration map, defined by $\left(\int \omega\right) s=\int_{\Delta^{n}} s^{*} \omega$, for any $n$-form $\omega$ and any smooth singular $n$-simplex $s: \Delta^{n} \rightarrow \mathscr{G}$.

Further, the canonical restriction map $\gamma: C_{0}(\mathscr{G}, \mathbb{R}) \rightarrow C_{\infty}(\mathscr{G}, \mathbb{R})\left(C_{0}(\mathscr{G}, \mathbb{R})\right.$ denotes the usual continuous singular co-chain complex of $\mathscr{G})$ induces isomorphism in cohomology.

Proof. Since the map $t:(\mathscr{G}, e) \rightarrow(\mathscr{G}, e)$ (where $e$ is the identity of $\mathscr{G})$ induces -1 at the tangent $T_{e}(\mathscr{G}) \approx \mathscr{L}$ level and, for any $g_{0} \in \mathscr{G}$, the map $\operatorname{Int}\left(g_{0}\right):(\mathscr{G}, e) \rightarrow(\mathscr{G}, e)$, defined by $\operatorname{Int}\left(g_{0}\right) g=g_{0} g g_{0}^{-1}$, induces the map $\operatorname{Ad}\left(g_{0}\right): \mathscr{L} \rightarrow \mathscr{L}$, the commutativity of the diagram follows easily. Caution! if we take all the left-invariant forms, instead of biinvariant forms, in the above diagram, it is no more commutative in general.

The assertion, that $\gamma$ induces isomorphism in cohomology, follows easily from the usual sheaf theoretic argument [W], if we observe that $\mathscr{G}$ (by assumption) is paracompact and is locally smoothly contractible (i.e., there exists a local smooth contraction).

In the above diagram, $\bar{t}$ is a co-chain map and so is $\int$ (by Stokes' theorem), hence any $\omega \in C(\mathscr{L})^{\mathscr{L}}$ is closed. (In fact, it can be easily seen that any $\omega \in C(\mathscr{L})^{\mathscr{L}}$, for arbitrary Lie algebra $\mathscr{L}$, is closed.) (This remark is due to M. S. Raghunathan.)

3. Corollary. Let $\omega \in C^{n}(\mathscr{L})^{\mathscr{L}}$ (be a cocycle) then the cohomology class $\left[\int \omega\right] \in H^{n}(\mathscr{G}, \mathbb{R})$ (we identify $H(\mathscr{G}, \mathbb{R})$ with the cohomology of the complex $C_{\infty}(\mathscr{G}, \mathbb{R})$ under the canonical restriction map $\gamma)$ transforms according to $(-1)^{n}$ under $t^{*}\left(t^{*}\right.$ is the induced map on cohomology by $t$ ).

An element $x \in H^{n}(\mathscr{G}, \mathbb{R})$ is said to be representable by a bi-invariant form if there exists a cocycle $\omega \in C^{n}(\mathscr{L})^{\mathscr{L}}$ such that $\left[\int \omega\right]=x$, as elements in $H^{n}(\mathscr{G}, \mathbb{R})$.

4. Remarks. (a) One important class of Lie groups (modelled on Fréchet spaces) is the Gauge group $\mathscr{G}(P)$, associated to a principal $G$-bundle (in the $C^{\infty}$-category) 
$P \rightarrow X$ (where $G$ is a finite dimensional Lie group and $X$ is a finite dimensional smooth manifold), defined as the group of $G$-equivariant smooth automorphisms of $P$, which cover the identity map of $X$. One can also take various Sobolev completions of $\mathscr{G}(P)$. See, e.g., [MV], [U].

(b) Another important class of groups, for which the above lemma is true (with appropriate definitions of the associated Lie algebra $\mathscr{L}$, integration map $\int$ and $\left.C_{\infty}(\mathscr{G}, \mathbb{R})\right)$, is the standard unitary form of Kac-Moody Groups/C. One particular example, of these (standard unitary form of) Kac-Moody groups, is one dimensional central extension of the loop group $\Omega(G)$, where $G$ is a finite-dimensional, compact, connected, simply-connected, simple Lie group and $\Omega(G)$ denotes the set of all the (unbased) loops: $S^{1} \rightarrow G$ with finite Fourier series. (Although these KacMoody groups are not smooth in any "reasonable sense," still Lemma (2) holds for them, as can be seen from $[\mathrm{Ku} ; \S 1]$.)

5. Lemma. Let $\mathscr{G}$ be any path-connected topological group. Fix a field $F$. Assume that $H^{i}(\mathscr{G}, F)$ is finite dimensional, for all $i$. Then the inversion map $t: \mathscr{G} \rightarrow \mathscr{G}$ induces the map -1 (multiplication by -1) on $H^{+}(\mathscr{G}, F) / H^{+}(\mathscr{G}, F)^{\cdot} \cdot H^{+}(\mathscr{G}, F)$, where $H^{+}(\mathscr{G}, F)$ denotes $\sum_{i>0} H^{i}(\mathscr{G}, F)$.

Proof. Consider the maps $\theta: \mathscr{G} \rightarrow \mathscr{G} \times \mathscr{G}$ and $m: \mathscr{G} \times \mathscr{G} \rightarrow \mathscr{G}$, defined by $\theta(g)=\left(g, g^{-1}\right)$ and $m(g, h)=g h$, for $g, h \in \mathscr{G}$. Of course, the induced map $m^{*}: H^{*}(\mathscr{G}) \rightarrow H^{*}(\mathscr{G}) \otimes H^{*}(\mathscr{G})$ satisfies, for any $x \in H^{+}(\mathscr{G}), m^{*}(x)=x \otimes 1+1 \otimes x+\sum_{i} x_{i} \otimes y_{i}$, for some (possibly empty) $x_{i}, y_{i} \in H^{+}(\mathscr{G})$. Also, the induced map $\theta^{*}: H^{*}(\mathscr{G}) \otimes H^{*}(\mathscr{G}) \rightarrow H^{*}(\mathscr{G})$ is given by $\theta^{*}(x \otimes y)=x \cdot t^{*}(y)(\cdot$ denotes the cup-product $)$.

Since $m \theta=e$, we get that $\theta^{*} m^{*}(x)=0$, for all $x \in H^{+}(\mathscr{G})$, i.e., $x+t^{*}(x)+\sum_{i} x_{i} \cdot t^{*}\left(y_{i}\right)$ $=0$. This proves the lemma.

6. Corollary. If $F$ is a field of characteristic $\neq 2$ and if $\mathscr{G}$ is as in the above lemma then (from complete reducibility) we can find a space of indecomposables, i.e., a graded section $\pi$ of the canonical projection: $H^{+}(\mathscr{G}) \rightarrow H^{+}(\mathscr{G}, F) / H^{+}(\mathscr{G}, F) \cdot H^{+}(\mathscr{G}, F)$, such that $t^{*} \pi(\bar{x})=-\pi(\bar{x})$, for any $\bar{x} \in H^{+}(\mathscr{G}) / H^{+}(\mathscr{G}) \cdot H^{+}(\mathscr{G})$.

We make the following

7. Definition. An element $x \in H^{*}(\mathscr{G}, F)$ is said to be primitive-like/ $F$ (respectively anti primitive-like $/ F)$ if $t^{*}(x)=-x$ (respectively $t^{*}(x)=x$ ).

Clearly, primitive elements are primitive-like.

Putting all these together, we get the following

8. Theorem. Let $\mathscr{G}$ be a path-connected topological group with all its Betti numbers $/ \mathbb{Q}$ being finite such that Lemma (2) holds for $\mathscr{G}$ (e.g., any connected Lie modelled on a Fréchet space with finite Betti numbers $/ \mathbb{Q}$, in particular, identity component of the gauge groups and also the standard unitary form of Kac-Moody groups).

Let $x \in H^{n}(G, \mathbb{R})$ be representable by a bi-invariant form. Then

(1) If $n$ is even, $x$ is anti primitive-like element.

(2) If $n$ is odd, $x$ is primitive-like element.

Further, there always exist primitive-like elements $\left\{x_{i}^{d(i)}\right\}_{i=1,2, \ldots}$, such that $x_{i}^{d(i)} \in H^{d(i)}(\mathscr{G}, \mathbb{R})$ and $H^{*}(\mathscr{G}, \mathbb{R})$ is freely generated (in the graded sense), as an 
algebra, by $\left\{x_{i}^{d(i)}\right\}_{i=1,2, \ldots}$. Of course, $\operatorname{dim} .\left(\Pi_{n}(\mathscr{G}) \underset{\mathbb{Z}}{\bigotimes} \mathbb{R}\right)=\#\{i: d(i)=n\}$, since $\sum_{n} \operatorname{Hom}_{\mathbb{Z}}\left(\Pi_{n}(\mathscr{G}), \mathbb{R}\right) \approx H^{+}(\mathscr{G}, \mathbb{R}) / H^{+}(\mathscr{G}, \mathbb{R}) \cdot H^{+}(\mathscr{G}, \mathbb{R})$, and the isomorphism is a graded vector space isomorphism. $\left(\Pi_{n}(\mathscr{G})\right.$ denotes the $n$-th homotopy group of $\mathscr{G}$.)

9. Corollary. With assumptions, as in the above theorem, on $\mathscr{G}$, let $x \in H^{2 d}(\mathscr{G}, \mathbb{R})$ with $d>0$. If $x$ is indecomposable, i.e., $x \notin H^{+}(\mathscr{G}, \mathbb{R}) \cdot H^{+}(\mathscr{G}, \mathbb{R})$, then $x$ cannot be represented by bi-invariant forms. In particular, if there exists a $d$ such that $\Pi_{2 d}(\mathscr{G}) \bigotimes_{\mathbb{Z}} \mathbb{R} \neq 0$, then there is a $x \in H^{2 d}(\mathscr{G}, \mathbb{R})$ which cannot be represented by biinvariant forms.

10. Examples. (a) Based loop groups $\Omega_{e}(G)$ ( $G$ is a finite dimensional, compact, connected, simply-connected Lie group). As is well known, $H^{*}\left(\Omega_{e}(G), \mathbb{R}\right)$ is generated by $\left\{x_{1}, \ldots, x_{l}\right\}$, where all the $x_{i}^{\prime}$ 's are of even degree. Hence, by the above corollary, none of the generators $x_{i}$ can be represented by bi-invariant forms, where as it is known that any class in $H^{*}\left(\Omega_{e}(G), \mathbb{R}\right)$ can be represented by a left-invariant form.

(b) Standard unitary form of Kac-Moody groups. It is known that for the standard unitary form $K$ of the Kac-Moody group $G$, associated to any indecomposable $l \times l$ generalized Cartan matrix $A$, except in the case when it is finite dimensional or $l=2$, we have $\sum_{n \geq 1} \Pi_{2 n}(K) \underset{\mathbb{Z}}{\bigotimes} \mathbb{R} \neq 0$. This follows from $[\mathrm{Ku}$; Theorem 3.8] together with [GS; Theorem 2] and also from [K; $\$ 2.6]$. Hence for any such $K$ (i.e., except when it is finite dimensional or $l=2$ ), there always exist classes in $H^{*}(K, \mathbb{R})$ which cannot be represented by bi-invariant forms. In contrast, all the cohomology classes can be represented by left-invariant forms. See [Ku; Theorem 1.6]. Hence the canonical inclusion $C\left(\mathbf{g}^{1}\right)^{\mathbf{g}^{1}} \hookrightarrow C\left(\mathbf{g}^{1}\right)$ does not induce surjection in cohomology, where $\mathbf{g}^{1}$ is the Kac-Moody Lie algebra/C (or more precisely its commutator sub-algebra) associated to the group $K$.

(c) Identity component $\mathscr{G}^{0}(P)$ of the gauge group $\mathscr{G}(P)$ (associated to a principal $G$ bundle $P \rightarrow X$, with $G$ a compact connected Lie group). Take a $\mathbb{R}$-basis $\left\{x_{j}^{n(j)}\right\}_{1 \leq 1 \leq k}$ of $H_{*}(X, \mathbb{R})$ (degree $\left.x_{j}^{n(j)}=n(j)\right)$ and let $\left\{c_{1}^{m(1)}, \ldots, c_{l}^{m(l)}\right\}$ be a set of free algebra generators of $H^{*}(B G, \mathbb{R})$ ( $m(i)$ 's are necessarily even). Then, by rational homotopy theory, $H^{*}\left(\mathscr{G}^{0}(P), \mathbb{R}\right)$ is a free (in the graded sense) algebra on a set of generators $\left\{\psi_{i, j}\right\}_{\text {only those } 1 \leq 1 \leq l \text { and } 1 \leq 1 \leq k \text { satisfyng } m(l)-n(1)>1}$. Moreover, the degree of $\psi_{i, j}=$ $m(i)-n(j)-1$. (A proof of this, in a particular case, is given in [AB; $\S 2]$.)

Hence any $\psi_{i, j}$, such that $n(j)$ is odd, cannot be represented by bi-invariant forms. In contrast, it is known that any $\psi_{i, j}$ with $m(i)>2 n(j)$ can, indeed, be represented by a left-invariant form, at least in the case when $G=U(l)$. (This was proved by Quillen in his lectures at M.I.T. during 1984-1985.)

Acknowledgement. My sincere thanks are due to Prof. D. Quillen for his very interesting course on gauge theory and related topics at M.I.T., which motivated the question (partially) answered in this paper.

\section{References}

[AB] Atiyah, M. F., Bott, R.: The Yang-Mills equations over Riemann surfaces. Philos. Trans. R. Soc. Lond. A308, 523-615 (1982)

[GS] Gutkin, E., Slodowy, P.: Cohomologie des variétés de drapeaux infinies. C.R. Acad. Sci. Paris 296, $625-627(1983)$ 
[K] Kac, V. G.: Constructing groups associated to infinite-dimensional Lie algebras. MSRI (SpringerVerlag) publications vol. 4 on 'Infinite dimensional groups with applications' 167-216 (1985)

[Ku] Kumar, S.: Rational homotopy theory of flag varieties associated to Kac-Moody groups. MSRI publications vol. 4 (same as in $[\mathrm{K}]$ ), 233-273

[M] Milnor, J.: Remarks on infinite-dimensional Lie groups. Relativity, groups and topology II, DeWitt, B. S. Stora, R. (eds.). Les Houches, 1009-1057 (1983)

[MV] Mitter, P. K., Viallet, C. M.: On the bundle of connections and the gauge orbit manifold in YangMills theory. Commun. Math. Phys. 79, 457-472 (1981)

[U] Uhlenbeck, K.: Connections with $L^{p}$ bounds on curvature. Commun. Math. Phys. 83, 31-42 (1982)

[W] Warner, F. W.: Foundations of differentiable manifolds and Lie-groups. New York: Scott, Foresman 1971

Communicated by A. Jaffe

Received July 12, 1985; in revised form March 21, 1986 
